\title{
AFM Measurements and Tip Characterization of Nanoparticles with Different Shapes
}

\author{
Roberto Bellotti $^{1}$ D $\cdot$ Gian Bartolo Picotto $^{1}$ (D) Luigi Ribotta $^{1,2}$ (D)
}

Received: 30 October 2021 / Revised: 7 January 2022 / Accepted: 13 January 2022 / Published online: 21 February 2022

(c) The Author(s) 2022

\begin{abstract}
Unambiguous identification of the measurement methodologies is fundamental to reduce the uncertainty and support traceability of particle shape and size at the nanoscale. In this work, the critical aspects in atomic force microscopy measurements, that is, drawbacks on sample preparation, instrumental parameters, image pre-processing, size reconstruction, and tip enlargement, are discussed in reference to quantitative dimensional measurements on different kinds of nanoparticles (inorganic and biological) with different shapes (spherical, cylindrical, complex geometry). Once the cross-section profile is extracted, top-height measurements on isolated nanoparticles of any shape can be achieved with sub-nanometer accuracy. Lateral resolution is affected by the pixel size and shape of the probe, causing dilation in the atomic force microscopy image. For the reconstruction of critical sizes of inorganic non-spherical nanoparticles, a geometric approach that considers the nominal shape because of the synthesis conditions is presented and discussed.
\end{abstract}

\section{Article Highlights}

- Methodologies for quantitative dimensional measurements on nanoparticles with different geometry by atomic force microscopy (AFM) are presented.

- Height measurements on isolated spherical and rod-shaped nanoparticles are extracted as cross-section top-height.

- Lateral measurements can be extracted by considering the tip dilation and nominal nanoparticle shape and size.

Keywords Nanoparticles (NPs) - Atomic force microscopy (AFM) - Size reconstruction $\cdot$ Spherical shape $\cdot$ Complex shape $\cdot$ AFM probe $\cdot$ Uncertainty

\section{Introduction}

Nanomaterials and nanotechnology play a crucial role in many products available on the global market, such as materials for energy and catalysis, cosmetic products, food, and packaging. These products are often produced with engineered nanoparticles (NPs), whose shape and size impart special functionalities.

Luigi Ribotta

1.ribotta@inrim.it

1 Istituto Nazionale di Ricerca Metrologica (INRiM), Strada delle Cacce 91, 10135 Torino, Italy

2 Politecnico di Torino, Corso Duca degli Abruzzi 24, 10129 Torino, Italy
At present, NPs and nanomaterial sizes are challenging to quantitatively measure, and nanometrology helps the manufacturing industry by developing new measurement methods. Low-cost reference materials are continuously being developed to characterize the 3D morphology at the nanoscale.

Atomic force microscopy (AFM) is a technique that allows the 3D characterization of various kinds of samples (conductive, insulators, inorganic, organic, biological, etc.) and operates in air or liquid. Given its versatility, AFM can be coupled with other techniques by using specialized hardware and types of probes (e.g., scanning thermal microscopy, Kelvin probe force microscopy, and infrared nanospectroscopy). AFM and various hyphenated techniques permit analysis in various fields, such as polymer chemistry and physics, surface chemistry, cell biology, semiconductor science, and metrology. 
In AFM measurements, height can be achieved with subnanometer accuracy and high resolution, whereas the lateral resolution is affected by the shape of the AFM probe. Therefore, various methods to build the morphology of the probe tip have been proposed. One of these methods is the use of electron microscopes, but related research demonstrates that the precise 3D morphology of the tip is hard to obtain because of the different experimental conditions and probe-sample dilation effects [1].

In situ probe shape reconstruction is preferable. One method is the estimation of the probe shape by imaging an unknown tip characterizer (blind reconstruction [BR] algorithms) [2,3], but the main drawback is the large amount of calculation [4]. In addressing this issue, another method is the calibration of the tip morphology by scanning a known tip characterizer [5]. Sharply pointed features are commercially available, but these characterizers lack traceability, which can lead to damage of tips [6]. Over the years, several physical artifacts for tip characterization have been presented, including nanospheres [7] and cylindrical nanostructures $[8,9]$.

The AFM topography results from the dilation of the sample shape, probe shape, and tip-sample-substrate interactions. An AFM image is commonly referred to as a 3D representation, although it is more referred to as a $2.5 \mathrm{D}$ reconstruction. The topography is a mapping of the heights given by a $2 \mathrm{D}$ array of numbers, which correspond to the deflection of the cantilever as the tip scans the sample surface. Therefore, a 3D reconstruction is identified, albeit limited by the tip geometry, which is not complete but is limited only to the exposed surface, and it does not consider the portion of the sample in contact with the substrate.

Tip-sample and tip-substrate interactions refer to elasticity, whereas sample-substrate deformation refers to plasticity. In describing these interactions, several models of contact mechanics are used, as shown in Sect. 4.2. Notably, these models strictly depend on the interacting geometries of the bodies.

NPs can assume different shapes, depending on the physi$\mathrm{cal} / \mathrm{chemical}$ interactions that regulate fabrication. The most popular shape is spherical, which can be easily obtained for energetically favorable reasons. Several reference materials based on spherical NPs, supplied in mono and/or multimodal dispersed suspensions, can be accurately measured, as demonstrated in several interlaboratory comparisons [10-12].

AFM measurements on non-spherical NPs are challenging because complex geometries emphasize the limits caused by the finite probe shape. New methodologies for traceable quantitative measurement sizes are required; these methods must be easily translated for the measurement of bulk nanostructures of various shapes. In this study, the geometric approaches implemented for studying the sizes of shape-controlled anatase NPs with different shapes (bipyramids and nanosheets) $[13,14]$ are reported.

\section{Samples}

\subsection{Sample Preparation}

Nanoparticles and nanostructures, such as biological molecules (e.g., DNA) or viruses, to be analyzed by AFM are deposited onto an ultra-flat substrate to permit high-resolution height and thickness measurements.

Muscovite mica is a commonly used substrate because it is atomically flat, with a layer height of $(0.37 \pm 0.02) \mathrm{nm}$ [15]. It is also easily cleaned by removing the top layer using adhesive tape, making sample preparation easy because its crystal structure consists of silica tetrahedral sheets weakly bonded to one another.

Muscovite is negatively charged, and is therefore quite hydrophilic. In addition, based on the sample to be analyzed, mica can be used as it is functionalized with 3-aminopropyltriethoxysilane (APTES) or poly-L-lysine to change the surface charge.

Different protocols can be used in the preparation of NP samples. Based on the intrinsic nature of the nanostructure (interparticle and particle-solvent interactions), different deposition techniques (e.g., drop evaporation, spin coating, and dip coating) are used. Moreover, characterization must be considered. Based on the type of analysis (i.e., top-height or lateral pitch), different concentrations of suspensions can be prepared.

\subsection{Nanoparticles}

In this work, we present the dimensional characterization of different kinds of nanoparticles, including bio-plant viruses (TMVs) and inorganic spherical (gold and silica) and nonspherical $\left(\mathrm{TiO}_{2}\right.$ anatase bipyramids and nanosheets) NPs.

For each NP, the analyzed measurand is different; for spherical and rod-shaped NPs, the diameter is measured, whereas for complex geometry NPs, characteristic critical sizes are reconstructed on the basis of the non-spherical shape. Consequently, the protocols for the preparation of samples with isolated NPs are different.

TMV is a single-stranded RNA virus that infects tobacco and Solanaceae plants, causing characteristic patterns, such as mosaic-like mottling and discoloration, on the infected leaves. It has a rod-like appearance, which has a length of approximately $300 \mathrm{~nm}$ and a stable diameter of $18 \mathrm{~nm}$ based on X-ray diffraction studies [16].

TMV is prepared by CNR-IPSP [9], inoculating the virus into the leaves, crushing the symptomatic leaves, and purifying them through a series of phosphate buffer extractions and 
centrifugations. After several dilutions with ultrapure water, a drop of viral suspension is deposited on freshly cleaved mica and allowed to air evaporate.

Spherical NPs are gold, and they have silica reference materials, namely, RM8012 by NIST [17] and ERM-FD304 by IRMM [18]. These NPs are deposited by applying a few drops of colloidal dispersion onto a mica surface previously functionalized with a $0.1 \% \mathrm{w} / \mathrm{v}$ aqueous poly-L-lysine solution; the sample is then incubated in a closed chamber to limit solvent evaporation, rinsed several times with filtered deionized water, and dried under nitrogen flow [12].

Complex geometry NPs are fabricated by Università degli Studi di Torino by tuning the hydrothermal synthesis parameters [14, 19], and obtaining $\mathrm{TiO}_{2}$ anatase NPs with two different shapes (bipyramids and nanosheets), which are mostly used for their photocatalytic properties. Samples for AFM imaging are made by depositing a drop of a $3 \mathrm{mg} \mathrm{L}^{-1}$ suspension of NPs onto a freshly cleaved mica support.

Notably, the topographies for each sample are registered in different areas of the mica support (usually three areas in the center and 5-6 areas at the borders). One sample for each spherical NP type is analyzed, whereas three samples of TMV, three samples of bipyramids, and three samples of nanosheets are imaged.

\section{Instrumentation and Measurements}

A metrological atomic force microscope (mAFM) is used to perform the measurements [20]. It is a custom-made instrument with a commercial AFM head arranged on a samplemoving mechanical structure that utilizes interferometers to control the relative tip-sample movements, thereby ensuring direct traceability to the meter unit.

Considering that the isolated NPs lie on the substrates via weak adhesion, measurements are conducted in a non-contact mode in air using amplitude demodulation. The RMS amplitude is less than $1 \mathrm{~nm}$. Standard silicon probes, with a nominal tip radius of $8 \mathrm{~nm}$, a force constant of $5 \mathrm{~N} \mathrm{~m}^{-1}$, and a resonance frequency of $160 \mathrm{kHz}$, are used. Notably, about 100 particles should be imaged for good statistical analysis.

The setting of instrumental parameters is important to register a good AFM image for quantitative measurements. Based on the requested relative height accuracy and radius of the tip used, the number of pixels in the image should be adjusted. Moreover, the operator must optimize the scan speed based on the scan size, and measurements must be performed using the right cantilever oscillation amplitude set point and PID feedback parameters.

Notably, each topography reported in this study is centered on one single NP; thus, each image reports a single particle. Therefore, images with a resolution of $(512 \times 512)$ pixels and scan sizes ranging from 300 to $500 \mathrm{~nm}$ are recorded. The speed used is selected to let the tip register the scan size in 1.5-2 s and to allow the tip to register a good image without instrumental noise.

The critical aspects in image pre-processing include the leveling of the image because a tilt that is not actually present on the sample surface is found, but it depends on sample mounting not perfectly perpendicular to the AFM tip. Linear plane fit corrections can be applied to remove any artifacts of consequence. In the analysis of nanoparticles, first-order correction can cause errors because the leveling function attempts to fit a polynomial to the substrate and nanoparticles instead of just fitting to the substrate. Therefore, during the analysis of isolated NPs, rotating the inclined plane is preferable.

Other artifacts may be due to the presence of steps among subsequent scan lines, which occur when the tip scanning direction is reversed or when some tip contaminations are observed. Therefore, a line-wise offset correction is made to eliminate these steps.

\section{Size Reconstruction/Determination}

Traceability and dissemination of samples and reference materials at the nanoscale are of great interest, as shown by several European projects, such as 3DNano [21] and nPsize [22]. Thus, defining methodologies for the measurement of critical sizes of NPs by using AFM is important. Notably, critical sizes refer to dimensions that uniquely describe the shape and geometry of a NP.

Strategies for the determination of top-height, lateral size, and tip characterization are discussed in the following sections. Tip-tilting [23] or critical dimension AFM [24] are used to accurately measure the dimensions of vertical features, such as the sidewalls of patterned semiconductor lines or high aspect ratio. In this paper, we focus on the characterization of various kinds of NPs by using selected commercial tips.

\subsection{Spherical Inorganic NPs}

As reported in the American ASTM E2859-11 [25] and the NPL GPG 119 [26] guides, two methods can be used to measure the diameter of spherical nanoparticles: (i) height of isolated NPs dispersed onto a flat substrate and (ii) lateral distance of NPs in a closely packed monolayer arrangement. Considering that the second method could be a source of errors because of tip dilation and a perfect closely packed monolayer arrangement of nanoparticles can be difficult to achieve, the first method is preferable.

In this measure, once the cross section of the NPs is extracted (Fig. 1a), the average substrate baseline is 


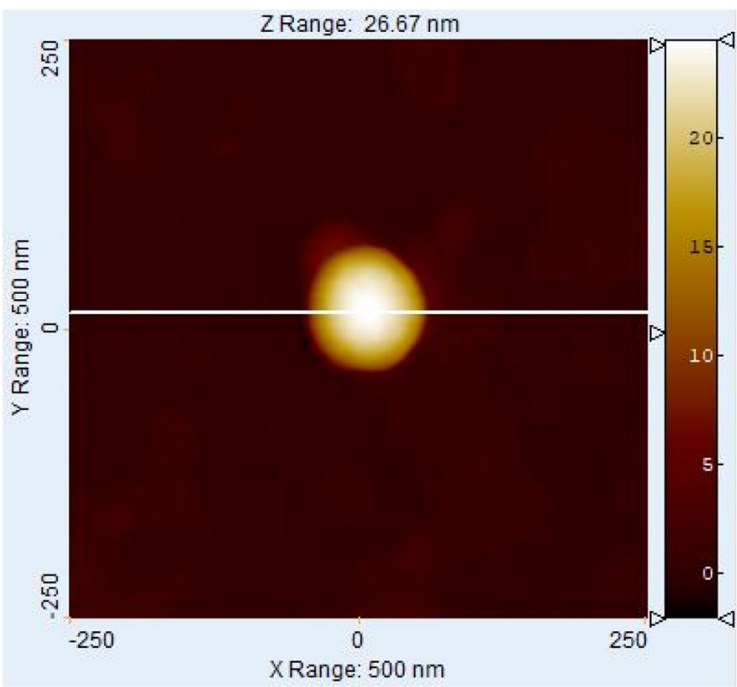

(a)

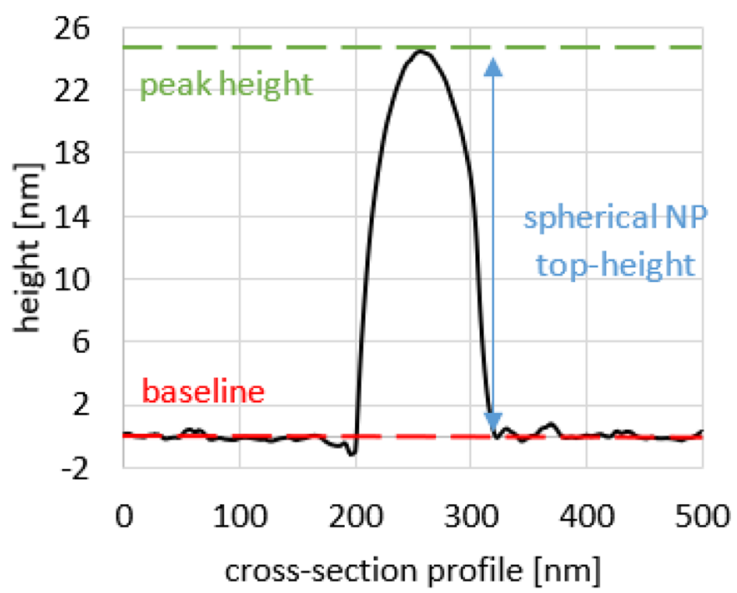

Fig. 1 a AFM topography of a gold spherical NP and $\mathbf{b}$ its cross-section profile. Measurement of the diameter of an isolated spherical NP as top-height is explained in $\mathbf{b}$

subtracted from the peak height to find the NP top-height (Fig. 1b).

The analyzed spherical NPs are gold and silica reference materials. Based on VIM, reference materials must be sufficiently homogeneous and stable with reference to specified properties, which have been established in the examination of nominal properties [27].

As an example of these measurements, a round robin between different national metrology institutes (NMIs) was conducted within the 3DNano project by using gold and silica spherical NPs to demonstrate the developed metrology capabilities to stakeholders and end users [12]. ERMFD304 by IRMM is a certified nanoparticle reference material (CRM), which is a "reference material, accompanied by documentation issued by an authoritative body and providing one or more specified property values with associated uncertainties and traceabilities, using valid procedures." [28].
RM8012 is a NIST reference material, which is a material issued by NIST with a report of investigation, depending on the organization wherein the materials may meet the ISO definition for a CRM.

These materials are used to perform instrument calibrations, verify the accuracy of specific measurements, and support the development of new measurement methods.

In determining the consistency of results from different participants, the degree of equivalence $E_{n}$ is calculated. The results are consistent if the absolute value of $E_{n}$ is smaller than 1. Moreover, the Birge ratio is evaluated, and for the consistency of results, this parameter must be less than a critical value depending on the number of participating laboratories. Based on these statistical tests, which are smaller than 1 for all participants, all the results are considered valid to calculate a reference value and associated uncertainty [12].

\subsection{Rod-Shape Bio-plant Viruses}

The same approach used for measuring the diameter of spherical NPs can be applied for measuring the diameter of the circle base of a cylindrical structure such as the TMV (Fig. 2).

As shown in Fig. 2c, the average TMV diameter value for 120 isolated viruses measured with MAFM is $(16.5 \pm 0.3)$ $\mathrm{nm}$, which is smaller than the nominal value of $18 \mathrm{~nm}$ by $\mathrm{X}$-ray fiber diffraction measurements.

The difference in results may be due to the interactions between the tip and the sample, the tip and the substrate, and the sample and the substrate. This assumption is first supported by the elastic moduli of various materials, which differ in orders of magnitude. TMV is a soft material with Young's modulus $E=(1.0 \pm 0.2) \mathrm{GPa}$ [29], whereas the mica and silicon tip are hard materials $\left(E_{\text {mica }}=(190 \pm 20)\right.$ $\mathrm{GPa}$ [30] and $E_{\mathrm{Si}}=(170 \pm 5) \mathrm{GPa}$ [31]).

Tip-substrate deformation $\alpha_{\text {tip-sub }}$ is calculated by using the Hertzian model of contact mechanics [32] considering the spherical silicon tip apex with a radius of $8 \mathrm{~nm}$ and the planar mica substrate with a deformation of $(0.02 \pm 0.01)$ nm.

The adhesion interaction between TMV and mica is calculated by using the Johnson-Kendall-Roberts (JKR) [33], Derjaguin-Muller-Toporov (DMT) [34] and Maugis-Pollock (MP) [35] models (which describe the deformation between a sphere and a plane) and Chaudhury model (which is more consistent after considering the interaction between a cylinder and a plane). The calculated deformations $\alpha_{\text {sam-sub }}$ are equal to $(0.99 \pm 0.29) \mathrm{nm}$ by using the JKR model, $(0.46 \pm 0.13) \mathrm{nm}$ by DMT, $(1.81 \pm 0.52) \mathrm{nm}$ by MP, and $(0.79 \pm 0.23) \mathrm{nm}$ by Chaudhury. The Hertzian model considers a purely elastic interaction without the presence of any forces; the JKR theory considers compressive and 
(a)
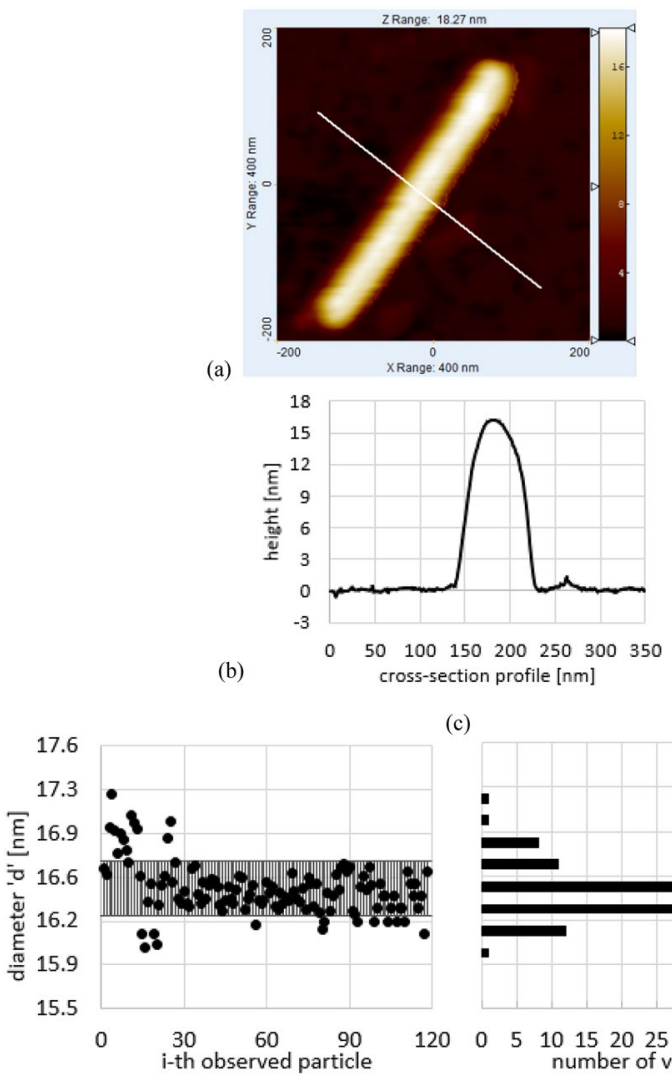

(c)

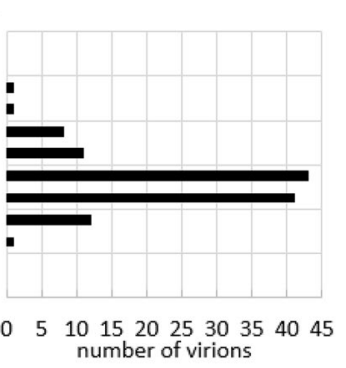

Fig. 2 a AFM topography of a TMV and $\mathbf{b}$ its cross-section profile. c Histogram of the TMV mean diameter measured as cross-section top-height

adhesive tractions inside the area of contact, whereas DMT considers only repulsive forces inside the contact area [36]. MP considers elastoplastic or full plastic contact [37]; thus, its contribution is higher compared with the other models.

The tip-sample term $\left(\alpha_{\text {tip-sam }}\right)$ considers the Hertzian interaction between a spherical tip and cylinder, and its value is $(0.35 \pm 0.10) \mathrm{nm}$.

Notably, the above-mentioned deformations and uncertainty are evaluated as the maximum error.

The overall deformation is calculated by $\alpha_{\mathrm{TOT}}=\alpha_{\text {tip-sam }}+$ $\alpha_{\text {sam-sub }}-\alpha_{\text {tip-sub }}=0.35+0.79-0.02 \sim 1.1 \mathrm{~nm}$; thus, the TMV diameter is calculated by the sum of the mean diameter from mAFM measurements, and the total deformation is $17.6 \mathrm{~nm}$.

Considering that the diameter of the TMV is stable once deposited onto the mica substrate, it can be used as a reference dimension for AFM tip calibration [8], and we develop a geometric approach to evaluate tip dilation.

Given the known circular shape and size of the base of the rod virus (Fig. 3a), we calculate the semi-chord of the circle by $s=\sqrt{t\left(2 r_{\mathrm{TMV}}-t\right)}$, where $t$ is the difference
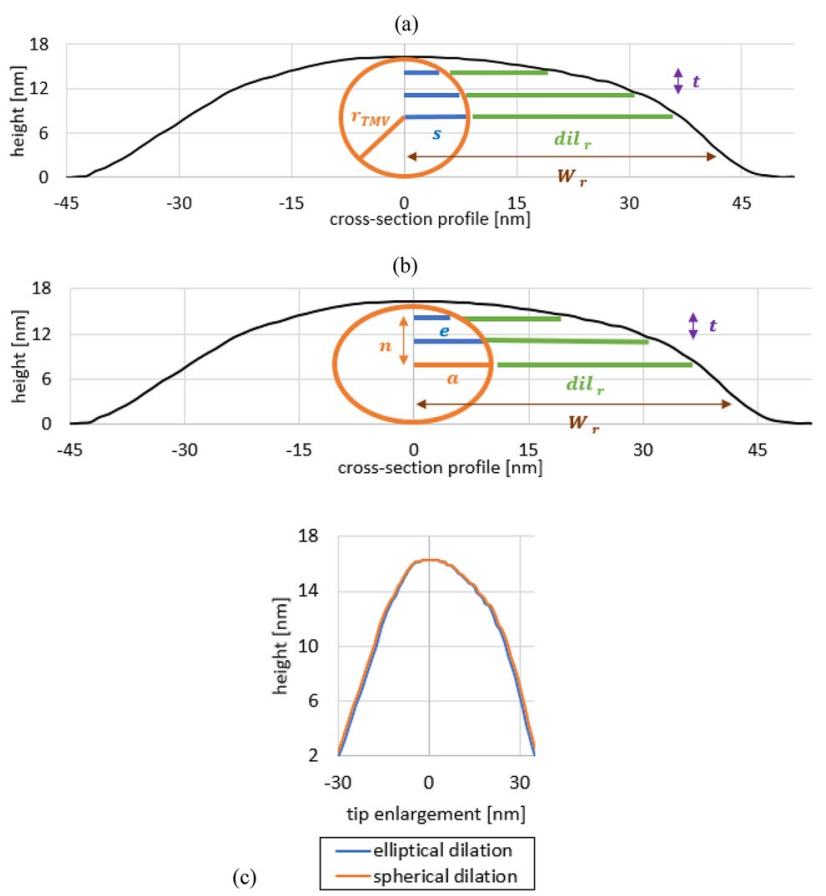

Fig. 3 a Spherical geometric approach to determine the tip dilation "dil" from the TMV cross-section profile. b Not-to-scale sketch illustrating the elliptical geometric approach to determine the tip dilation "dil" from the TMV cross-section profile. c Tip enlargement profile comparison between spherical and elliptical methods

between the top circle height to the height of the chord at which the dilation is calculated.

Notably, the shape of the virus affects the accuracy of tip characterization. In real measurements, the shape of the virus is not ideally cylindrical because of (i) the sample-substrate interaction $\alpha_{\text {sam-sub }}$ and (ii) the AFM probing force that affects the tip-sample interaction $\alpha_{\text {tip-sam }}$. Considering these interactions, an elliptical geometry of the TMV cross-section is reported (Fig. 3b); the elliptical semi-chord is calculated by $e=\frac{a}{n} \sqrt{t(2 n-t)}$, where $t$ is the difference between the top ellipse height to the height of the chord at which the dilation is calculated, and $a$ and $n$ are the major and minor semiaxis of the ellipse, respectively.

Figure $3 \mathrm{a}, \mathrm{b}$ show the right dilation $d i l_{r}$, which is calculated by the difference between the right width $W_{\mathrm{r}}$ and the semi-chord s/e based on the model used.

The same calculation is performed for the left side of the profile to calculate $d i l_{l}$, and the sum of the right and left dilation is calculated to obtain the overall tip dilation profile. Figure $3 \mathrm{c}$ reports the comparison between the spherical and elliptical models, showing a lateral difference of 3\%. Notably, the cylinder is primarily deformed in the part that is in contact with the substrate, but we expect that for the upper semi-cylindrical section of the virus, slightly deformed by 
the tip-sample elastic interaction, the approximation with a circle is consistent.

\subsection{Complex Geometry of Inorganic NPs}

Most of the industrial NPs have irregular shapes; thus, defining new measurement methodologies for a robust 3D reconstruction of shapes and sizes is important. Complex geometric NP sizes are difficult to analyze by AFM because of the finite size of the tip, which dilates the imaged sample.

Using anatase NPs directly as a physical artifact, we develop geometric approaches for the simultaneous reconstruction of (i) critical sizes based on the crystal intrinsic characteristic and (ii) the dilation profile based on the tip shape.

A characteristic of $\mathrm{TiO}_{2}$ anatase crystals is the interfacial angle $\vartheta=(68.3 \pm 0.3)^{\circ}$, which is measured by X-ray diffraction [38]. By tuning the synthesis parameters, anatase crystals may assume different shapes. The bipyramids have an elongated truncated bipyramid shape with a base square, whereas nanosheets are squashed bipyramids (Fig. 4).

The geometric approach developed for the analysis of bipyramid CSs is based on two assumptions: (i) the presence of a square base bipyramid caused by the anatase crystal and (ii) the shape isotropy of the tip apex.

Figure 5a shows a cross-section profile along the NP minor axis, in which the bipyramid base is sketched, and
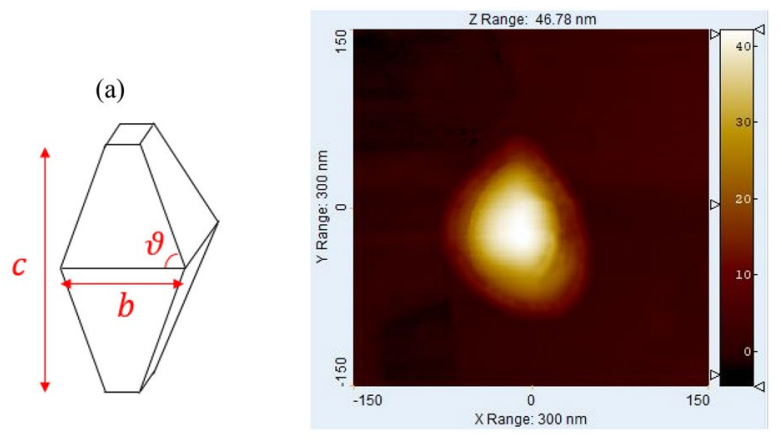

(b)
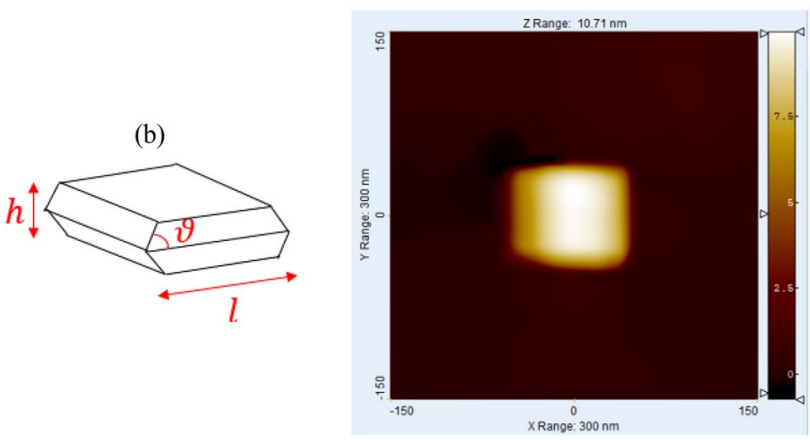

Fig. 4 a 3D sketch of a bipyramid and an AFM topography imaged with mAFM. b 3D sketch of a nanosheet and an AFM topography imaged with $\mathrm{mAFM}$

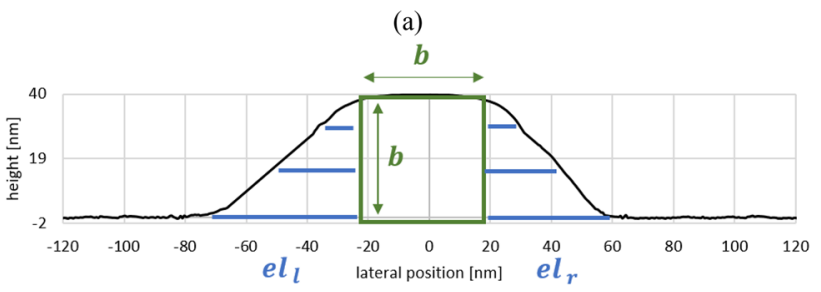

(b)

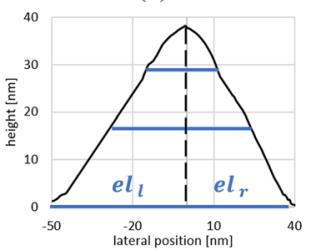

(c)

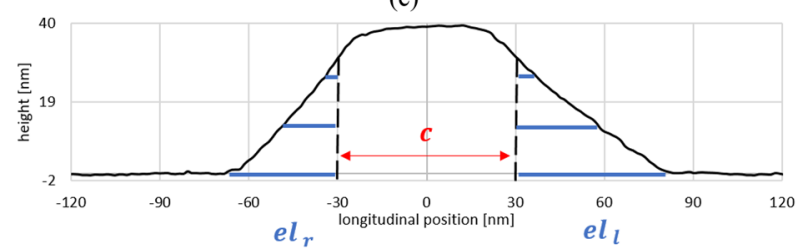

Fig. 5 Not-to-scale sketches illustrating the geometric approach to determine the bipyramid critical sizes. a Cross-section profile along the minor bipyramid axis reporting the square base with side length " $b$ " and the left/right tip enlargement $e l_{l}$ and $e l_{r}$. b Tip dilation profile obtained by deleting the square of Fig. 5a. c Cross-section profile along the major bipyramid axis reporting the determination of the critical size " $c$."

the square side $b$ is defined as the thickness and lateral length at the center of the profile. The blue segments outside the square describe the dilation based on the tip, and a tip dilation profile can be extracted on the basis of the union of the right and left enlargement (Fig. 5b).

Figure $5 \mathrm{c}$ shows the profile along the major axis of the bipyramid, and the size $c$ is obtained by deleting the enlargement segment $e l_{l}$ and $e l_{r}$.

The AFM measurement results are reported in Fig. 6, and the sizes are $b=(43.2 \pm 3.4) \mathrm{nm}$ and $c=(58.2 \pm 5.2) \mathrm{nm}$. The dispersion is narrow and monomodal.

Nanosheet geometric analysis is based on its geometry (a truncated tetragonal bipyramidal shape squashed along the [001] crystalline axis) and on the anatase interfacial angle $\vartheta=(68.3 \pm 0.3)^{\circ}$. By using this angle, the cross-section profile is sliced into various segments of known length $l_{\text {top }}+f_{i}$, describing the nanosheet lateral dimension $l$ and the tip dilation $e$ (Fig. 7).

The distributions of the CSs of the nanosheets are monomodal, but the height $h$ has a narrow dispersion, and the lateral dimension is more dispersed because of the growth of the crystals during synthesis (Fig. 8). The results from AFM measurements are $h=(9.3 \pm 1.4) \mathrm{nm}$ and $l=(75.4 \pm 25.8)$ $\mathrm{nm}$ and are consistent with those obtained from transmission-mode scanning electron microscopy [14]. 
(a)

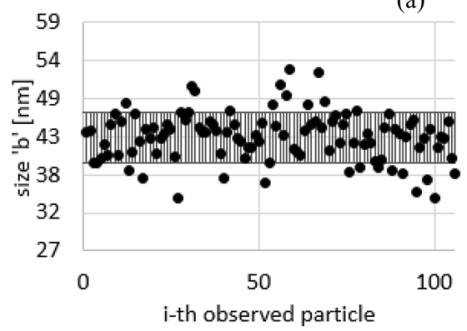

(b)

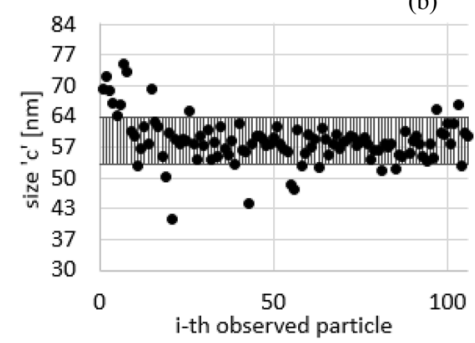

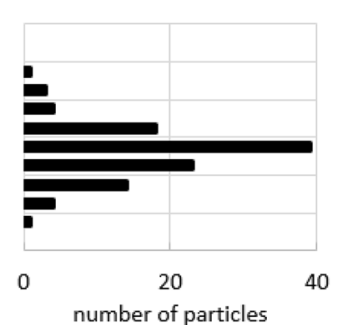

number of particles

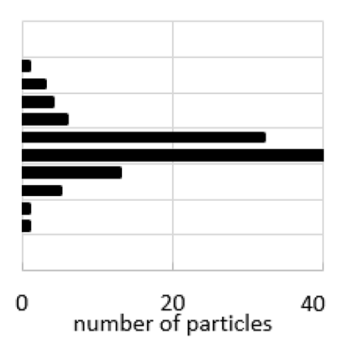

Fig. 6 a Repeatability plot and histogram of the bipyramid size " $b$ ". b Repeatability plot and histogram of the bipyramid size " $c$ ". The vertical lines in the repeatability plots refer to the standard deviation of the mean of the 106 analyzed particles

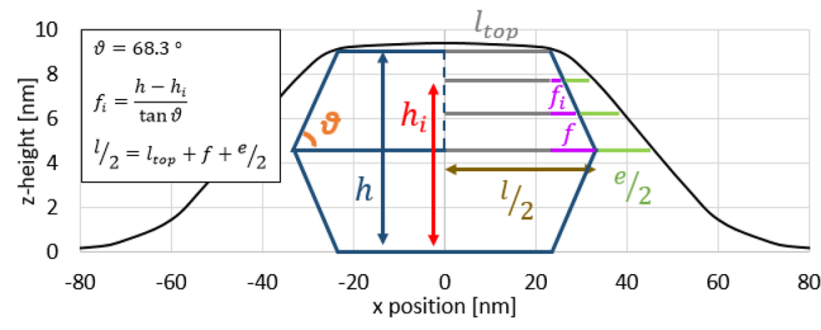

Fig. 7 Sketch illustrating the geometric approach to determine the nanosheet critical sizes. Note that the same calculation is repeated for the left side of the NP

\section{Tip dilation}

AFM lateral resolution is affected by (i) the pixel size, (ii) tip shape, and (iii) thermo-mechanical noise. Pixelization affects resolution because it cannot resolve features smaller than the pixel size of the image.

When the AFM tip interacts with the sample, the surface height at the apex of the tip is recorded, whereas the true surface of the sample may lie at a different location. Dilation arises from the finite size and geometry of the tip, and it is pronounced when the sizes of the sample and tip are similar.

Two main methods can be used in the reconstruction of the AFM tip shape, including the use of "BR" models or the scanning of a "tip characterizer."

BR methods allow the estimation of the tip shape from an AFM image. Villarrubia [2] was the first to use a

(a)
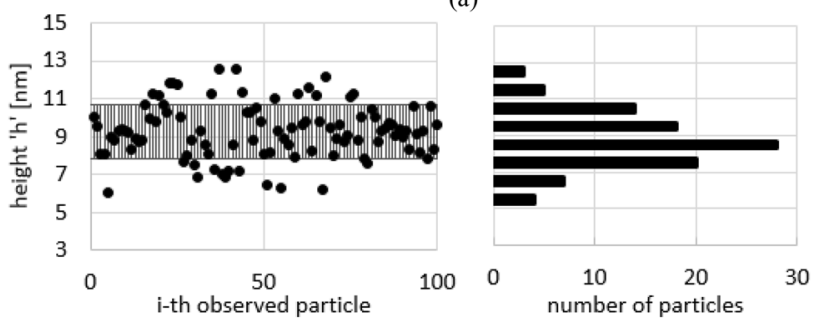

(b)
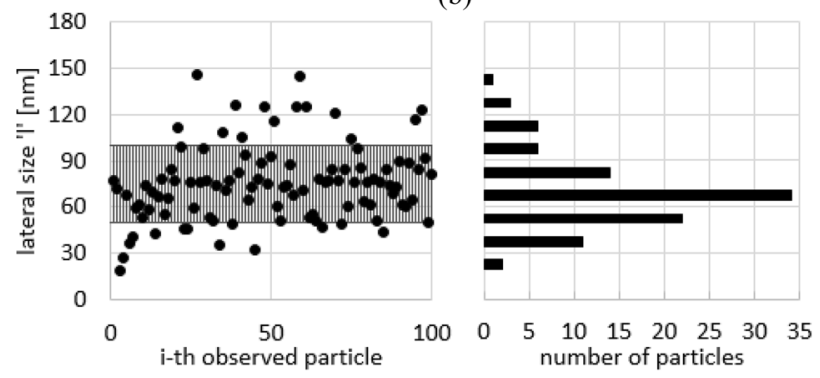

Fig. 8 a Repeatability plot and histogram of the nanosheet height " $h$ ". b Repeatability plot and histogram of the nanosheet lateral size " $l$." The vertical lines in the repeatability plots refer to the standard deviation of the mean of the 100 analyzed particles

mathematical morphology for tip characterization. Afterward, several studies were conducted to enhance the calculation algorithm, containing noise caused by the image [39] and proposing reasonable parameter settings [3].

For the estimation of the tip shape by using the BR method implemented on MATLAB by Flater et al. [3], a "square pillar" with a flat top is selected as the initial estimate. After the initialization of parameters, the algorithm iterates through all possible contact points to estimate the best tip shape. Optimizing the "tip matrix size" and the "threshold value" parameters is important to obtain the best estimate for the tip shape. The tip matrix size must have the same lateral dimension as the largest object imaged in the topography. The threshold parameter establishes a tolerated level of inconsistency between the image and tip estimate. These values are proportional to the image $Z$-range; if they are too low, then the tip reconstruction is dominated by the image noise, whereas if they are too high, then no features on the image are sharp enough to allow the modification of the initial "square pillar" geometry.

BR algorithms reconstruct a deconvoluted image to identify the deepest penetration of the tip, but if the tip geometry is not appropriate or the sample does not contain sharp features, then errors may occur during the reconstruction of tip and sample geometries. Scanning a known tip characterizer is advantageous because the sample sizes can be reconstructed by correcting the sampled image with the "true" nominal shape. On the contrary, the BR method reveals an 
upper boundary of the tip geometry only, but in reality, tip characterizers are not infinitely sharp [40].

Several tip characterizers are available in the market [41, 42], but in Sects. 4.2 and 4.3, we have presented biological nanostructures and non-spherical nanoparticles as potential tip characterizers. These samples have specific peculiarities; TMVs are low-cost nanostructures available worldwide, whereas bipyramids and nanosheets have peculiar geometries to be fully characterized as potential reference non-spherical nanoparticles [43].

The comparison on the estimation of the shape of a commercial tip between BR software, which is proposed by Flater et al. [3], and our geometric approach is shown in Fig. 9. These reconstructions refer to the analysis of the same bipyramid image.

In estimating the tip shape, the parameters set in the Flater software include (i) a tip matrix size of 100 pixels, (ii) a number of thresholds of 20 , and (iii) a threshold step
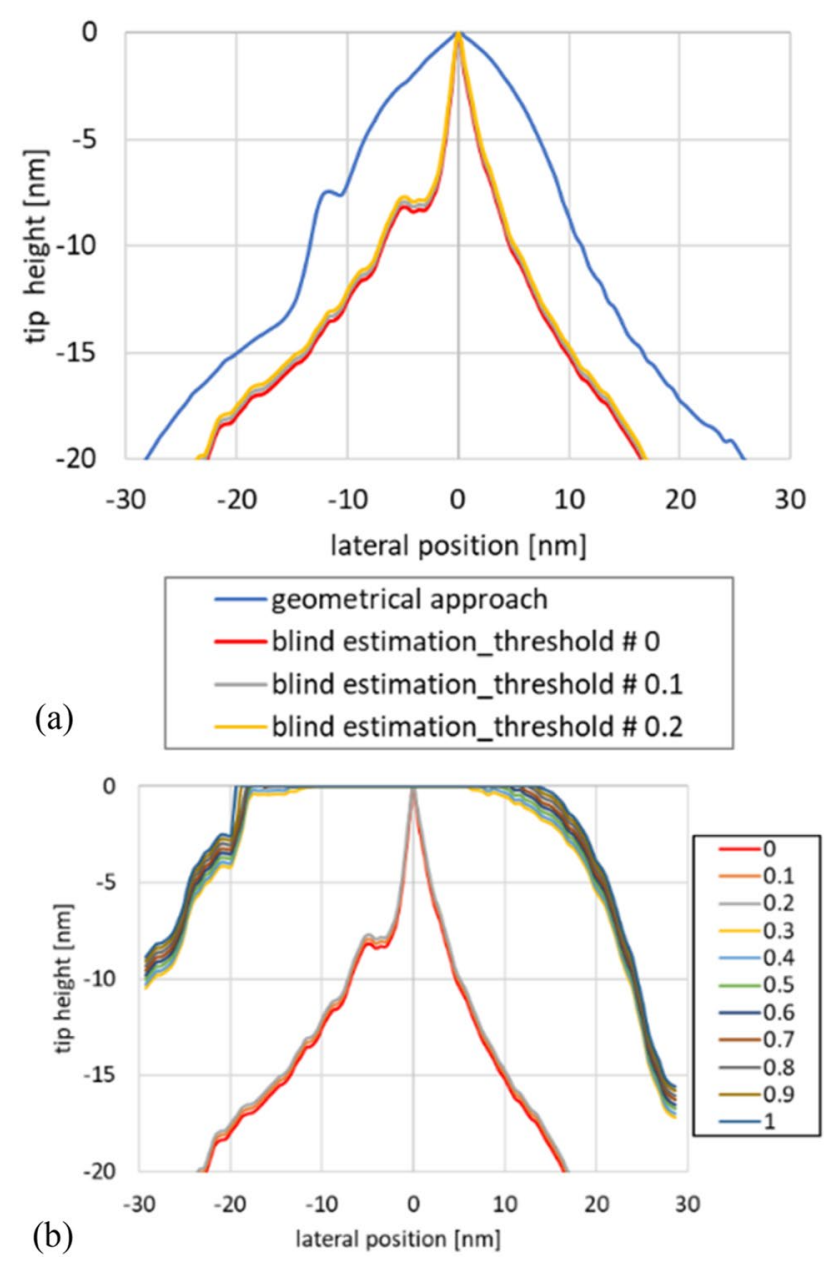

Fig. 9 a Comparison between "blind reconstruction" and "geometric approach" for tip shape estimation. b Tip shape estimation profiles after threshold refinement (in the legend, the refined threshold numbers are reported) size of $0.05 \mathrm{~nm}$. The tip shape is equal to $5 \%$ of the NP height, obtaining a threshold step size of $2.35 \mathrm{~nm}$. Then, a refinement of thresholds is made to obtain a threshold step size of $0.235 \mathrm{~nm}$. Figure 9 shows three profiles obtained with a smaller step size.

As shown in Fig. 9a, the BR method estimates a shape sharper than the shape obtained by our geometric approach (lateral difference of about 30\%), but the two methods analyze the same nanoparticle in a rigorous way because both methods reconstruct the same image artifact (small peak at the left side). Notably, BR software struggles during the erosion of the initial "square pillar" (Fig. 9b). For threshold numbers higher than 0.3 , the tip shape estimate is not significantly different from the original square pillar, whereas for smaller threshold numbers, tip reconstructions are dominated by image noise and tend to be unrealistically sharp in the absence of a more realistic reconstruction of the tip shape. The lateral offset (Fig. 9a) is due to the different reconstruction methods. Using our approach, a conical shape is reconstructed, and the outcome of the BR may transform from a square pillar-like shape to an unrealistic sharp shape.

Moreover, the BR method consumes a high computation time (the reconstruction takes about $24 \mathrm{~h}$ using a personal computer with 8 GB RAM and a $3.40-\mathrm{GHz}$ processor because of the high number of pixels and threshold for the estimation of the tip shape).

Therefore, based on tip shape analysis from AFM topographies centered on single NPs deposited onto flat substrates, the geometric approach is a suitable option. On the contrary, in the analysis of AFM images with a rougher substrate and several NPs, the BR method is recommended. Our geometric method is primarily developed to study NP critical sizes, following the reconstruction of the tip dilation profile.

\section{Uncertainty}

Providing measurement intervals is important to make quantitative measurements; the standard deviation provides the repeatability, whereas in uncertainty budgets, various sources of error are quantified. A measurement without uncertainty cannot be compared either with other measures or with reference values or legal limits.

Therefore, the main error sources in AFM top-height and lateral NP size measurements are reported in Ishikawa plots. These sources are used in the evaluation of uncertainty budgets on various measurands, which are described previously. For various samples, the subsequent combined standard uncertainties of the mean values of measurands are calculated: $4 \%$ for the gold NP diameter, $4 \%$ for the silica NP diameter, $4 \%$ for the top-height of the TMV, $4 \%$ for $b$ and $5 \%$ for $c$ bipyramid dimensions, $10 \%$ for the height $h$, and $5 \%$ for the lateral $l$ of the nanosheets. 


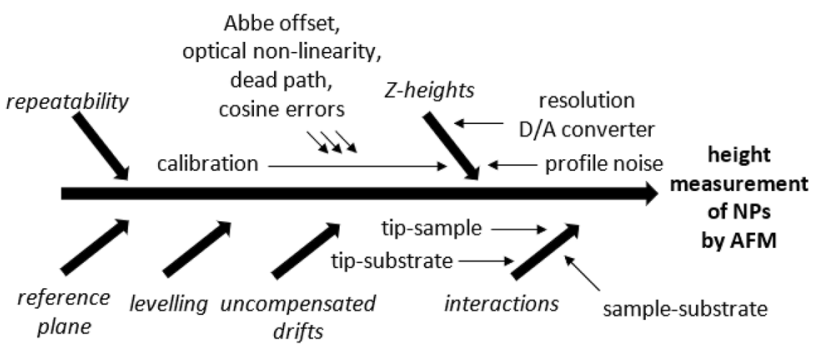

Fig. 10 Fishbone diagram reporting the uncertainty contributions that must be considered in NP height measurements from AFM images

Figure 10 shows the uncertainty sources for height measurements. In particular, these sources refer to the uncertainty of the particle diameter for NPs with spherical and rod shapes and height measurement for NPs with a non-spherical shape. These contributions are valid not only for the top-height of NPs, but also for step-height thickness measurements.

The uncertainty sources have different nature probably because of (i) instrumental configuration ("Z-heights" and "uncompensated drift" contributions) and (ii) the measurand and sample under analysis ("repeatability," "reference plane," "leveling," and "interaction" terms).

The "Z-height" considers (i) the calibration of the instrumental setup, (ii) the profile noise along the $X$-direction, and (iii) the resolution of the digital/analog converter.

The "uncompensated drifts" refer to mechanical drifts, which are evaluated as the straightness variation before and after line-wise correction.

The "repeatability" considers the standard deviation of the mean of the analyzed measurands.

The "reference plane" refers to the definition of the baseline (Fig. 1b).

The "leveling" of the substrate is evaluated through the variation of the height value by tilting the substrate orientation.

The "interaction" terms refer to the uncertainty related to the various models used for describing the elastic/elastoplastic interactions. For anatase NPs, tip-sample-substrate interactions are negligible because anatase NPs and tip are hard materials with a high elastic modulus.

The main contributions in the uncertainty evaluation of the mean diameter of inorganic spherical NPs are the repeatability of measurements, the uncompensated drifts, and the $Z$-calibration of the mAFM.

The major contribution of the uncertainty to the mean TMV circular base, which is evaluated as cross-sectional top-height, is the mAFM Z-calibration and the corrections of sample-substrate deformation.

The combined standard uncertainty for the square side $b$ is equal to $5 \%$, and the main contributions are due to the reference plane and Z-heights.
Figure 11 shows the Ishikawa plot for the reconstruction of lateral sizes of complex geometric NPs. The " $X$-size" term is due to (i) the image side and (ii) the pixel size based on the resolution and dimensions of the images and (iii) the resolution of the digital/analog converter.

The "tip" contribution considers (i) the dilation, which is evaluated by analyzing the lateral enlargement of the dilation profile measured by the same tip on subsequent images and (ii) the isotropy of the apex of the probe.

The main contributions of the uncertainty budget of the bipyramid lateral CS $c$ mean value are the uncertainty of $b$, the correction of tip dilation, and the $X$-size term.

Finally, all three contributions shown in Fig. 11 (repeatability, $X$-size, and tip) are important in the evaluation of the uncertainty of the nanosheet side $l$.

\section{Conclusions}

Various techniques are used for 3D analysis at the nanoscale, such as modeling-supported scatterometry for CD metrology and 3D SEM reconstruction from a stereo pair. AFM is a widely used instrumental technique that directly provides a $2.5 \mathrm{D}$ reconstruction of nanostructures and nanoparticles, with further capabilities provided by CD-AFM developments.

In this study, isolated nanoparticles of different nature and shapes are analyzed with selected standard tips by using a metrological AFM to ensure direct traceability of dimensional measurements to the SI.

Height measurements can be achieved with sub-nanometer accuracy and high resolution. Once the cross-section of the NPs is extracted, the average substrate baseline is subtracted from the peak height to find the top-height of NPs.

The combined standard uncertainty of the mean diameter $d$ of spherical gold and silica reference material NPs with a nominal diameter of $30 \mathrm{~nm}$, which is evaluated as top-height, is equal to $0.9 \mathrm{~nm}+0.01 d$, as indicated by our calibration measurement capability (CMC). The uncertainty is given by

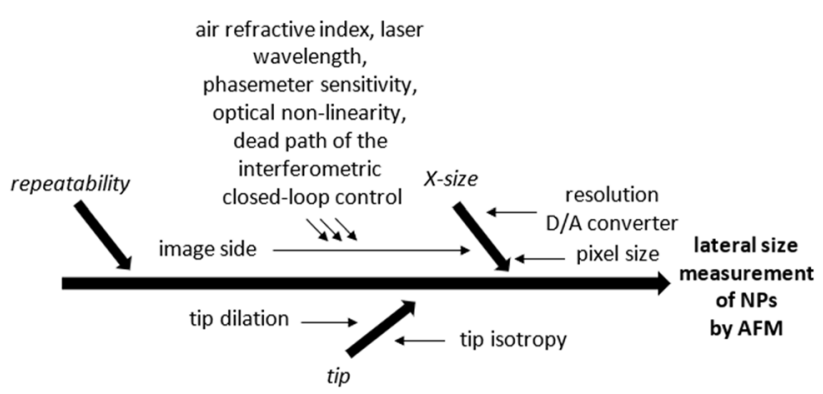

Fig. 11 Fishbone diagram reporting the uncertainty contributions to be considered in the measurement of NPs' lateral size from AFM images 
a constant term, which is primarily influenced by the repeatability of measurements and reference plane definition, and a proportional term, which is measured by the interferometric calibration of the $Z$-axis and sample leveling.

These measurements are conducted within the interlaboratory comparison organized within the EMPIR 3DNano project, and the values of all NMI participants agree with those reported by suppliers. Moreover, our CMC is consistent with the capabilities of other NMIs for spherical NPs of similar nominal size.

Based on X-ray diffraction measurements, TMV is a rodshaped virus with a stable diameter of $18 \mathrm{~nm}$. Considering that the TMV top-height measured by AFM is $16.5 \mathrm{~nm}$, tip-sample-substrate interactions are evaluated by using different models of contact mechanics. The total deformation is approximately $1.1 \mathrm{~nm}$; thus, the difference between X-ray and top-height diameter reduces to approximately $0.4 \mathrm{~nm}$.

The combined standard uncertainty on the mean TMV circular base measured as top-height is slightly better than that from spherical NPs because of the better repeatability of measurements. However, the significant contribution of sample-substrate elastoplastic adhesion hinders the application of this kind of measurement.

Once the base of the cylindrical nanostructure is fully characterized, we reconstruct the enlargement caused by the tip by lateral measurements, assuming the circular/elliptical cross-section of the virus.

Determining the size of non-spherical NPs by AFM is a non-trivial challenge because of the finite shape of the tip and the complex geometry of the NP. In this study, an approach based on intrinsic characteristics of the NP crystalline structure is presented.

The geometric approach developed for the analysis of bipyramid CSs (namely, $b$ and $c$ ) is based on two assumptions: (1) the presence of a square base bipyramid caused by the anatase crystal and (2) the isotropy of the tip shape at its apex. The square side measured as top-height on 106 bipyramids is $b=(43.2 \pm 3.4) \mathrm{nm}$, and its combined standard uncertainty of the mean is primarily affected by the definition of the reference plane. The CS $c=(58.2 \pm 5.2) \mathrm{nm}$ describes the size that lies on the major bipyramid axis, and its combined standard uncertainty is influenced by the uncertainty of $b$, the correction of tip dilation, and the $X$-size term.

Nanosheet geometric analysis is based on the anatase interfacial angle, by which "slicing" the cross-section profile into various segments of known length is possible, describing the nanosheet lateral dimension and tip dilation.

The nanosheet thickness measured on 100 particles is $h=(9.3 \pm 1.4) \mathrm{nm}$, and its uncertainty is primarily affected by the definition of the reference plane and instrumental calibration. The lateral size is $l=(75.4 \pm 25.8) \mathrm{nm}$, and this size is strongly affected by the repeatability and tip dilation.
Therefore, the uncertainties on complex geometric NPs are greater than those of spherical NPs because of the large distribution caused by the synthetic process. Lateral measurements with smaller uncertainties are obtained by the inspection of line width standard with CD-tips.

Notably, all the mean values of the measurands on the non-spherical NPs are consistent with those performed on the same batch by transmission-mode scanning electron microscopy.

Therefore, our geometric approach allows the quantitative analysis of selected measurands of isolated bipyramids and nanosheets in a robust, repeatable, and fast way. This geometrical model can be easily adapted to other non-spherical geometries once the nominal dimensional characteristics based on the synthetic process of non-spherical NPs are known. Further developments on this geometric approach will provide a comprehensive analysis of tip dilation in mixed samples containing several isolated spherical and non-spherical geometric NPs.

Author's contributions All authors contributed to the study conception and design. Material preparation and data collection were performed by Luigi Ribotta and Roberto Bellotti, while data analysis was performed by Luigi Ribotta and Gian Bartolo Picotto. The first draft of the manuscript was written by Luigi Ribotta and Gian Bartolo Picotto commented on previous versions of the manuscript. All authors read and approved the final manuscript.

Funding No funding was received for conducting this study.

Data availability The research data are available upon request to the corresponding author.

\section{Declarations}

Conflict of interest All authors certify that they have no affiliations with or involvement in any organization or entity with any financial interest or non-financial interest in the subject matter or materials discussed in this manuscript.

Open Access This article is licensed under a Creative Commons Attribution 4.0 International License, which permits use, sharing, adaptation, distribution and reproduction in any medium or format, as long as you give appropriate credit to the original author(s) and the source, provide a link to the Creative Commons licence, and indicate if changes were made. The images or other third party material in this article are included in the article's Creative Commons licence, unless indicated otherwise in a credit line to the material. If material is not included in the article's Creative Commons licence and your intended use is not permitted by statutory regulation or exceeds the permitted use, you will need to obtain permission directly from the copyright holder. To view a copy of this licence, visit http://creativecommons.org/licenses/by/4.0/. 


\section{References}

1. Postek MT (1994) Critical issues in scanning electron microscope metrology. J Res Natl Inst Stand Technol 99:641

2. Villarrubia JS (1997) Algorithms for scanned probe microscope image simulation, surface reconstruction, and tip estimation. J Res Natl Inst Stand Technol 102(4):425-4544. https://doi.org/10.6028/ jres.102.030

3. Flater EE, Zacharakis-Jutz GE, Dumba BA, White IA, Clifford CA (2014) Towards easy and reliable AFM tip shape determination using blind tip reconstruction. Ultramicroscopy 146:130-143. https://doi.org/10.1016/j.ultramic.2013.06.022

4. Wan J, Xu L, Wu S, Hu X (2014) Investigation on blind tip reconstruction errors caused by sample features. Sensors (Basel) 14(12):23159-23175. https://doi.org/10.3390/s141223159

5. Keller D, Franke FS (1993) Envelope reconstruction of probe microscope images. Surf Sci 294:409-419. https://doi.org/10. 1016/0039-6028(93)90126-5

6. Slattery AD, Blanch AJ, Quinton JS, Gibson CT (2013) Efficient attachment of carbon nanotubes to conventional and highfrequency AFM probes enhanced by electron beam processes. Nanotechnology 24:235705. https://doi.org/10.1088/0957-4484/ 24/23/235705

7. Ramirez-Aguilar KA, Rowlen KL (1998) Tip characterization from AFM images of nanometric spherical particles. Langmuir 14(9):2562-2566. https://doi.org/10.1021/la971277o

8. Trinh $\mathrm{M}-\mathrm{H}$, Odorico $\mathrm{M}$, Bellanger L, Jacquemond M, Parot P, Pellequer J-L (2011) Tobacco mosaic virus as an AFM tip calibrator. J Mol Recognit 24:503-510. https://doi.org/10.1002/jmr.1118

9. Picotto GB, Vallino M, Ribotta L (2020) Tip-sample characterization in the AFM study of a rod-shaped nanostructure. Meas Sci Technol 31:084001. https://doi.org/10.1088/1361-6501/ab7bc2

10. Meli F, Klein T, Buhr E, Frase CG, Gleber G, Krumrey M, Duta A, Duta S, Korpelainen V, Bellotti R, Picotto GB (2012) Traceable size determination of nanoparticles, a comparison among European metrology institutes. Meas Sci Technol 23:125005. https://doi.org/10.1088/0957-0233/23/12/125005

11. Lin H-L, Fu W-E, Weng H-F, Misumi I, Sugawara K, Gonda S, Takahashi K, Takahata K, Ehara K, Takatsuji T, Fujimoto T, Salas J, Dirscherl K, Garnaes J, Damasceno J, de Oliveira JCV, Emanuele E, Picotto GB, Kim CS, Cho SJ, Motzkus C, Meli F, Gao S, Shi Y, Liu J, Jämting K, Catchpoole HJ, Lawn MA, Herrmann J, Coleman VA, Adlem L, Kruger OA, Buajarern J, Buhr E, Danzebrink H-U, Krumrey M, Bosse H (2019) Nanoparticle characterization-supplementary comparison on nanoparticle size. Metrologia 56:04004. https://doi.org/10.1088/0026-1394/56/1A/ 04004

12. Sebaihi N, Pétry J, Koops R, Valtr M, Klapetek P, Dai G, Hausotte T, Klöpzig U, Wu Y, Korpelainen V (2019) Good practice guide for dimensional metrology at the nanometer scale in general and for using the developed reference standards and methodologies in particular, EMPIR 15SIB09 Traceable three-dimensional nanometrology (3DNano) project. https://www.ptb.de/emrp/3738.html

13. Mino L, Pellegrino F, Rades S, Radnik J, Hodoroaba VD, Spoto G, Maurino V, Martra G (2018) Beyond shape engineering of $\mathrm{TiO}_{2}$ nanoparticles: post-synthesis treatment dependence of surface hydration, hydroxylation, Lewis acidity and photocatalytic activity of $\mathrm{TiO}_{2}$ anatase nanoparticles with dominant 001 or 101 facets. ACS Applied Nano Mater 1(9):5355-5365. https://doi.org/ 10.1021/acsanm.8b01477

14. Pellegrino F, Sordello F, Mino L, Minero C, Hodoroaba V-D, Martra G, Maurino V (2019) Formic acid photoreforming for hydrogen production on shape-controlled anatase $\mathrm{TiO}_{2}$ nanoparticles: assessment of the role of fluorides, $\{101\} /\{001\}$ surfaces ratio, and platinization. ACS Catal 9:6692-6697. https://doi.org/ 10.1021/acscatal.9b01861

15. Xu K, Cao P, Heath JR (2010) Graphene visualizes the first water adlayers on mica at ambient conditions. Science 329(5996):11881191. https://doi.org/10.1126/science. 1192907

16. Namba K, Stubbs G (1986) Structure of tobacco mosaic virus at $3.6 \AA$ resolution: implications for assembly. Science. https://doi. org/10.1126/science. 3952490

17. RM 8012, Gold nanoparticles-nominal $30 \mathrm{~nm}$ diameter, National Institute of Standards and Technology OR National Bureau of Standards, U.S. Department of Commerce, Gaithersburg. https:// www-s.nist.gov/srmors/view_detail.cfm?srm $=8012$

18. European Commission Science Hub. https://ec.europa.eu/jrc/en/ science-update/irmm-releases-erm-fd304-nanoparticle-referencematerial

19. Pellegrino F, Isopescu R, Pellutiè L, Sordello F, Rossi AM, Ortel E, Martra G, Hodoroaba V-D, Maurino V (2020) Machine learning approach for elucidating and predicting the role of synthesis parameters on the shape and size of $\mathrm{TiO}_{2}$ nanoparticles. Sci Rep 10:18910. https://doi.org/10.1038/s41598-020-75967-w

20. Picotto GB, Pisani M (2001) A sample scanning system with nanometric accuracy for quantitative SPM measurements. Ultramicroscopy 86(1-2):247-254. https://doi.org/10.1016/s03043991(00)00112-1

21. EMPIR 15 SIB 09 project "Traceable three-dimensional nanometrology (3DNano)". https://www.ptb.de/emrp/15sib09-home.html

22. EMPIR 17NRM04 project "Improved traceability chain of nanoparticle size measurements (nPSize)". https://www.bam.de/Conte nt/EN/Projects/nPSize/npsize.html

23. Kizu R, Misumi I, Hirai A, Kinoshita K, Gonda S (2018) Development of a metrological atomic force microscope with a tiptilting mechanism for 3D nanometrology. Meas Sci Technol 29(7):075005. https://doi.org/10.1088/1361-6501/aabe1a

24. Dai G, Hahm K, Bosse H, Dixson R (2017) Comparison of line width calibration using critical dimension atomic force microscopes between PTB and NIST. Meas Sci Technol 28:065010. https://doi.org/10.1088/1361-6501/aa665b

25. ASTM E2859-11(2017) Standard guide for size measurement of nanoparticles using atomic force microscopy, American Society for Testing and Materials (ASTM)

26. Boyd RD, Cuenat A, Meli F, Klein T, Frase CG, Gleber G, Krumrey M, Duta A, Duta S, Hogstrom R, Prieto E (2011) Good practice guide for the determination of the size and size distribution of spherical nanoparticle samples, National Physical Laboratory

27. JCGM 200:2012, International vocabulary of metrology-basic and general concepts and associated terms (VIM 3rd edition), definition 5.13

28. JCGM 200:2012, International vocabulary of metrology-basic and general concepts and associated terms (VIM 3rd edition), definition 5.14

29. Zhao Y, Ge Z, Fang J (2008) Elastic modulus of viral nanotubes. Phys Rev E 78:031914. https://doi.org/10.1103/PhysRevE.78. 031914

30. Castellanos-Gomez A, Poot M, Amor-Amorós A, Steele GA, van der Zant HSJ, Agrait N, Rubio-Bollinger G (2012) Mechanical properties of freely suspended atomically thin dielectric layers of mica. Nano Res 5:550-557. https://doi.org/10.1007/ s12274-012-0240-3

31. Hopcroft MA, Nix WD, Kenny TW (2010) What is the Young's modulus of silicon? J Microelectromech Syst 19:2. https://doi.org/ 10.1109/JMEMS.2009.2039697

32. Puttock MJ, Thwaite EG (1969) Elastic compression of spheres and cylinders at point and line contact. National Standards Laboratory Technical Paper No. 25 
33. Johnson KL, Kendall K, Roberts AD (1971) Surface energy and the contact of elastic solids. Proc R Soc Lond A 324:301-313. https://doi.org/10.1098/rspa.1971.0141

34. Derjaguin BV, Muller VM, Toporov YP (1975) Effect of contact deformations on the adhesion of particles. J Colloid Interface Sci 53(2):314-326. https://doi.org/10.1016/0021-9797(75)90018-1

35. Maugis D, Pollock HM (1984) Surface forces, deformation and adherence at metal microcontacts. Acta Metall 32(9):1323-1334. https://doi.org/10.1016/0001-6160(84)90078-6

36. Ciavarella M, Joe J, Papangelo A, Barber JR (2019) The role of adhesion in contact mechanics. J R Soc Interface 16:151. https:// doi.org/10.1098/rsif.2018.0738

37. Bloo ML, Haitjema H, Pril WO (1999) Deformation and wear of pyramidal, silicon-nitride AFM tips scanning micrometre-size features in contact mode. Measurement 25(3):203-211. https:// doi.org/10.1016/S0263-2241(99)00004-4

38. Yang HG, Sun CH, Qiao SZ, Zou J, Liu G, Smith SC, Cheng HM, Lu GQ (2008) Anatase $\mathrm{TiO}_{2}$ single crystals with a large percentage of reactive facets. Nature 453:638-641. https://doi. org/10.1038/nature06964

39. Todd BA, Eppell SJ (2001) A method to improve the quantitative analysis of SFM images at the nanoscale. Surf Sci 491(3):473483. https://doi.org/10.1016/S0039-6028(01)01313-9

40. Dai G, Xu L, Hahm K (2020) Accurate tip characterization in critical dimension atomic force microscopy. Meas Sci Technol 31:074011. https://doi.org/10.1088/1361-6501/ab7fd2

41. http://www.supracon.com/en/afmtip_characterizer.html. Accessed Sept 2021

42. Takenaka H, Hatayama M, Ito H, Ohchi T, Takano A, Kurosawa $\mathrm{S}$, Itoh $\mathrm{H}$, Ichimura S (2011) Development of $\mathrm{Si} / \mathrm{SiO}_{2}$ multilayer type AFM tip characterizers. J Surf Anal 17(3):264. https://doi. org/10.1384/jsa.17.264

43. Mansfeld U, Pellegrino F, Maurino V, Marguet S, Testard F, Tachè O, Hodoroaba V-D (2019) New reference material candidates for traceable size measurement of non-spherical nanoparticles. In: 18th European conference on applications of surface and interface analysis (ECASIA 19), Dresden, Germany

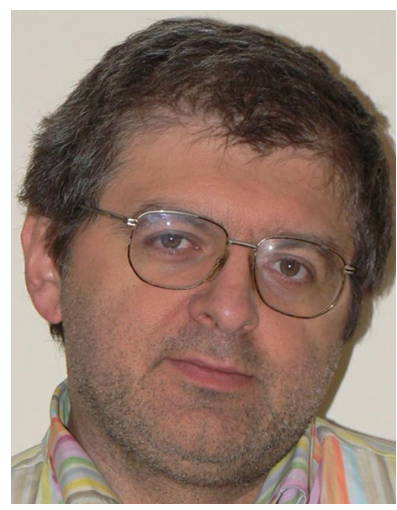

Roberto Bellotti is a technician who carries out both calibrations and measurements to support research in dimensional metrology. He deals with measurements ranging from the millimetric range, by using a $1 \mathrm{D}$ comparator equipped with an interferometric system, to the nanometric range, by using a metrological atomic force microscope and optical sensors coupled with interferometric systems.

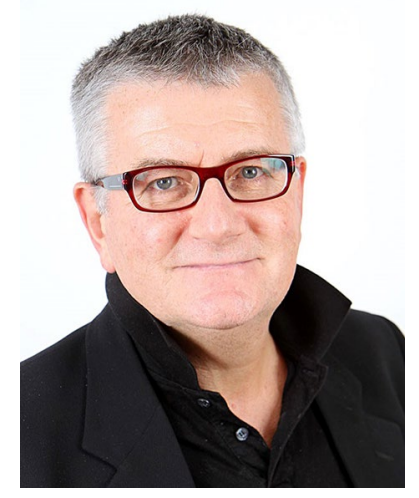

Gian Bartolo Picotto is an experienced length metrologist with a background on optical frequency standards and laser spectroscopy. Senior Researcher at the Istituto Nazionale di Ricerca Metrologica (INRiM), he has been responsible for the research on surface metrology, diameter, line and end standards. His main interests are in scanning probe microscopy, optical and stylus profilometry, and laser interferometry.

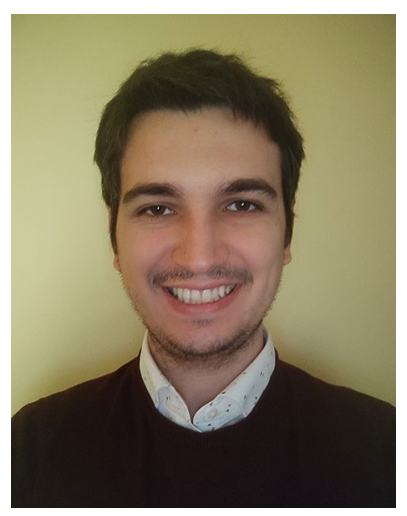

Luigi Ribotta is a PhD student in "Metrology" at the Politecnico di Torino/INRiM (XXXIV cycle), and his research focuses on the metrology of nanoparticles, in particular on 3D dimensional characterization by using a metrological atomic force microscope. Previously, at INRiM he carried out a two-year research fellow period concerning surface metrology, aimed at determining the correlation between the morphological and functional parameters on photovoltaic cells and lab-on-chip. 